Quim. Nova, Vol. 36, No. 4, 582-586, 2013

\title{
DESENVOLVIMENTO E VALIDAÇÃO DE UM MÉTODO ANALÍTICO POR ESPECTROFOTOMETRIA UV PARA QUANTIFICAÇÃO DE CARVEDILOL
}

\author{
Paola Aline Amarante Borba*, Manoela Klüppel Riekes, Rafael Nicolay Pereira e Hellen Karine Stulzer \\ Departamento de Ciências Farmacêuticas, Universidade Federal de Santa Catarina, 88040-900 Florianópolis - SC, Brasil \\ Debora Dalla Vecchia \\ Departamento de Ciências Farmacêuticas, Universidade Estadual de Ponta Grossa, 84030-900 Ponta Grossa - PR, Brasil
}

Recebido em 5/7/12; aceito em 22/10/12; publicado na web em 20/2/13

\begin{abstract}
DEVELOPMENT AND VALIDATION OF A UV SPECTROPHOTOMETRIC METHOD TO QUANTIFY CARVEDILOL. This paper reports the development and validation of a new analytical method using UV spectrophotometry to quantify carvedilol (CRV) in hydrophilic matrices and raw material. This method was shown to be linear, accurate, precise, robust and to have adequate limits of quantification and detection (LQ and LD, respectively), allowing its use in the dissolution test of hydrophilic matrices. The content of CRV determined through this method was compared with two previously validated methods based on the reference techniques of High Performance Liquid Chromatography (HPLC) and Potentiometric Titrations (PT). ANOVA confirmed the equivalence of these methods, showing no significant differences.
\end{abstract}

Keywords: carvedilol; UV spectrophotometry; validation.

\section{INTRODUÇÃO}

A hipertensão arterial é considerada um importante problema de saúde e uma das principais causas de morbidade e mortalidade na maioria dos países desenvolvidos do mundo. ${ }^{1,2}$ Sua prevalência mundial é de aproximadamente $30 \%$, sendo considerada um importante fator de risco para o acidente vascular cerebral, doença cardíaca isquêmica e insuficiência cardíaca. Neste contexto, a hipertensão arterial representa um elevado custo econômico-social sobre os sistemas de saúde, famílias e sociedade como um todo. ${ }^{2}$

O carvedilol (CRV), Figura 1, é um agente hipotensor antagonista não seletivo dos receptores $\beta$-adrenérgicos, que promove a diminuição da resistência vascular periférica através do bloqueio dos receptores adrenérgicos $\alpha 1{ }^{3,4}$ É amplamente utilizado no tratamento da hipertensão arterial sistêmica e controle de doenças cardiovasculares associadas, no intuito de reduzir a mortalidade cardiovascular em pacientes vítimas de infarto do miocárdio, a insuficiência cardíaca e a disfunção ventricular esquerda. ${ }^{5,6}$<smiles>COc1ccccc1OCCNCC(O)COc1cccc2[nH]c3ccccc3c12</smiles>

Figura 1. Estrutura química do CRV

O CRV apresenta-se na forma de pó cristalino de coloração branca ou quase branca e é caracterizado como uma base fraca praticamente insolúvel em água, com tempo de meia-vida de 6-10 h, requerendo duas administrações diárias..$^{7-9}$ Dessa forma, este fármaco possui baixa biodisponibilidade (em torno de $25 \%$ ) e significante metabolismo de primeira passagem. ${ }^{7-9}$ Tais características biofarmacêuticas tornam o CRV alvo de pesquisas e desenvolvimento de sistemas de liberação modificada, como os comprimidos de matrizes hidrofílicas. ${ }^{10}$

A quantificação de um fármaco em uma formulação e na sua

*e-mail: paola_borba@hotmail.com forma isolada representa um aspecto fundamental da garantia da qualidade de medicamentos. ${ }^{11} \mathrm{O}$ desenvolvimento e a validação de métodos analíticos têm como objetivo verificar se o fármaco se apresenta apropriado para a finalidade a que se destina e se esta é capaz de fornecer resultados confiáveis, que possam ser satisfatoriamente interpretados. ${ }^{12,13}$

A monografia oficial para o doseamento de CRV encontra-se descrita na Farmacopeia Britânica, ${ }^{14}$ contudo esta se aplica apenas à matéria-prima e se dá através da técnica titulométrica. As Farmacopeias Americana ${ }^{15}$ e Brasileira ${ }^{16}$ não contemplam a monografia para este fármaco.

A literatura apresenta poucos métodos referentes à determinação do CRV puro e em formulações farmacêuticas. ${ }^{17-19}$

Dentre as diferentes técnicas analíticas, a de cromatografia líquida de alta eficiência (CLAE) foi empregada tanto na determinação do teor do fármaco em comprimidos, acoplada a um detector de fluorescência, ${ }^{18}$ quanto no isolamento e identificação de impurezas/produtos de degradação do CRV em comprimidos matriciais, acoplado a um detector UV. ${ }^{17,20}$

A técnica espectrofotométrica na região do ultravioleta (UV), amplamente aplicada no doseamento de fármacos, ${ }^{21-23}$ é utilizada principalmente no setor de controle de qualidade de indústrias farmacêuticas, uma vez que cumpre requisitos indispensáveis na rotina laboratorial como rapidez, baixo custo operacional e elevada confiabilidade de resultados. ${ }^{13,24,25}$

Apesar de bastante vantajosa para a indústria farmacêutica, a literatura apresenta poucos artigos visando a determinação do CRV através da técnica espectrofotométrica na região do UV. ${ }^{19,26,27}$ Esta técnica foi relatada na determinação do teor de CRV em comprimidos $^{19,26}$ e em comprimidos combinados com outros agentes anti-hipertensivos, ${ }^{27}$ ambos utilizando o metanol como solvente. Entretanto, não há relatos na literatura de método analítico para a quantificação do CRV matéria-prima e em comprimidos matriciais hidrofílicos por espectrofotometria de absorção na região do ultravioleta empregando $\mathrm{HCl}$ 0,1 M como solvente. A técnica espectrofotométrica na região do UV apresenta-se como uma alternativa barata, rápida, de fácil utilização e interpretação de resultados, além de oferecer elevada confiabilidade dos resultados. ${ }^{12,13,24,25}$ Desse 
modo, este trabalho teve como objetivos o desenvolvimento e a validação de um método analítico para a quantificação do fármaco isolado e em comprimidos matriciais por espectrofotometria no ultravioleta, bem como a realização de um estudo comparativo dos resultados obtidos com duas técnicas de referência previamente validadas: titulometria potenciométrica (TP) ${ }^{27}$ e CLAE, ${ }^{20}$ ambos os métodos validados para o doseamento do fármaco matéria-prima $\mathrm{e}$ em comprimidos. Para a validação foram avaliados os parâmetros de especificidade, linearidade, limites de quantificação e de detecção, precisão, exatidão e robustez.

\section{PARTE EXPERIMENTAL}

\section{Materiais}

\section{Reagentes e amostras}

O CRV foi adquirido através da distribuidora DEG Importadora de Produtos Químicos LTDA (São Paulo, Brasil). O padrão de CRV é proveniente da Farmacopeia Brasileira. Os comprimidos matriciais de CRV foram desenvolvidos no laboratório de controle de qualidade da Universidade Estadual de Ponta Grossa, em parceria com a Indústria Farmacêutica Prati Donaduzzi Ltda. ${ }^{28}$ A água ultrapura foi obtida pelo sistema Millipore Milli-Q ${ }^{\circledR}$ (Millipore Corp., New Bedford, MA, USA).Os demais reagentes utilizados apresentavam grau analítico ou cromatográfico de acordo com a técnica empregada.

\section{Preparo da solução padrão}

A solução padrão contendo $500 \mu \mathrm{g} \mathrm{mL}^{-1}$ de $\mathrm{CRV}$ foi preparada solubilizando-se $25 \mathrm{mg}$ de CRV em pequena quantidade de álcool etílico ( $7 \mathrm{~mL}$ ), completando-se o volume do balão volumétrico de 50 $\mathrm{mL}$ com solução de $\mathrm{HCl}$ 0,1 M. Esta solução foi mantida à temperatura ambiente e protegida da luz.

\section{Preparo das amostras}

Dez comprimidos matriciais contendo $50 \mathrm{mg}$ de CRV foram pesados e triturados a pó fino, conforme preconizado na Farmacopeia Brasileira. ${ }^{16}$ Em seguida, quantidade equivalente a $50 \mathrm{mg}$ do fármaco foi pesada e solubilizada em quantidade mínima de etanol (14 mL), empregando uma solução de $\mathrm{HCl} 0,1 \mathrm{M}$ como diluente para obter a concentração final de $6 \mu \mathrm{g} \mathrm{mL}{ }^{-1}$. As soluções dos comprimidos matriciais foram filtradas em filtro quantitativo. Esta solução foi mantida à temperatura ambiente e protegida da luz.

\section{Equipamentos}

Espectrofotômetro UV/Vis (Varian, Cary 50 Bio). Sistema de cromatografia líquida de alta eficiência (Shimadzu LC -10 A, Japão) com detector UV/VIS SPD-10AVP e controlador SCL-10 AVP. Software Class VP ${ }^{\circledR}$. Coluna cromatográfica Luna C18 Phenomenex ${ }^{\circledR}$ com $250 \mathrm{~mm}$ de comprimento, 4,6 $\mathrm{mm}$ de d.i. e $5 \mu \mathrm{m}$ de tamanho de partícula. Pré-coluna C18 Phenomenex ${ }^{\circledR}$ com $4 \mathrm{~cm}$ de cumprimento e 3,0 mm de diâmetro.

\section{Validação do método analítico por espectrofotometria na região do UV}

O método foi validado conforme o preconizado na Resolução $\mathrm{RE} \mathrm{n}^{\circ} 899$, de 29/5/2003, e com as recomendações da ICH (The International Conference on Harmonisation of Technical Requirements for Registration of Pharmaceuticals for Human Use). ${ }^{12,29,30}$ Os parâmetros avaliados foram especificidade, linearidade e faixa, limites de quantificação (LQ) e detecção (LD), precisão, exatidão e robustez.

\section{Especificidade}

A especificidade do método foi avaliada através de análises comparativas entre as formulações placebo (sem o fármaco) e as contendo o CRV, realizadas em triplicata. Para tal, preparou-se uma solução do padrão na concentração de $6 \mu \mathrm{gL}^{-1}$ e uma solução do placebo, composta pelos excipientes que compõem a matriz do comprimido. A solução placebo foi preparada utilizando-se a concentração dos excipientes dentro da faixa normatizada na literatura. ${ }^{31}$ As soluções padrão e placebo foram filtradas em filtro quantitativo anteriormente às varreduras espectrais na faixa de 200-700 nm do UV-Visível (UV-VIS).

\section{Linearidade e faixa}

A linearidade foi determinada através da construção de seis curvas de calibração na faixa de $2-10 \mu \mathrm{g} \mathrm{mL} \mathrm{m}^{-1}$. Para a construção de cada curva de calibração foram preparadas cinco concentrações (2, 4, 6, 8 e $\left.10 \mu \mathrm{g} \mathrm{mL}^{-1}\right)$, a partir da solução padrão de CRV. Para tal, transferiram-se diferentes alíquotas da solução padrão de CRV na concentração de $500 \mu \mathrm{g} \mathrm{mL}^{-1}$ para um balão volumétrico de 50 $\mathrm{mL}$, completando o volume do balão com $\mathrm{HCl}$ 0,1 M. Estas foram analisadas em triplicata e a linearidade foi estimada por regressão linear. As absorbâncias foram quantificadas em triplicata a $240 \mathrm{~nm}$.

Limites de quantificação $(L Q)$ e detecção $(L D)$

Os limites de quantificação (LQ) e de detecção (LD) foram calculados a partir das curvas de calibração. Calculou-se LD e LQ a partir das equações $3.3 \sigma / \mathrm{S}$ e $10 \sigma / \mathrm{S}$, respectivamente, onde $\sigma$ é o desvio padrão da resposta e $\mathrm{S}$ é o coeficiente angular da curva. ${ }^{29,30}$

\section{Precisão}

A precisão intradia (repetibilidade) foi avaliada através de medições, em triplicata, de seis soluções de amostra na concentração de $6 \mu \mathrm{g} \mathrm{mL} \mathrm{m}^{-1}$, em diferentes períodos do mesmo dia (manhã, tarde e noite) sobre as mesmas condições experimentais. A precisão interdias (precisão intermediária) foi avaliada através dos resultados obtidos pelas análises das amostras na mesma concentração de $6 \mu \mathrm{g} \mathrm{mL}^{-1}$, em dias seguidos e distintos. O parâmetro de precisão foi avaliado para o CRV matéria-prima e em comprimidos matriciais.

\section{Exatidão}

Para determinação da exatidão, procedeu-se à adição de concentrações conhecidas do padrão de $\operatorname{CRV}\left(2,4\right.$ e $\left.8 \mu \mathrm{g} \mathrm{mL} \mathrm{m}^{-1}\right)$ à solução

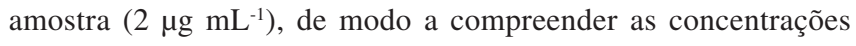
finais obtidas dentro da faixa de linearidade do método. Os valores de recuperação, expressos em porcentagem, foram determinados a partir das respostas analíticas obtidas das soluções finais em função da quantidade teórica de padrão adicionado.

\section{Robustez}

A robustez do método proposto foi determinada em triplicata, a partir de soluções de trabalho na concentração de $6 \mu \mathrm{g} \mathrm{mL}{ }^{-1}$, variando-se os comprimentos de onda $(238,240$ e $242 \mathrm{~nm})$, os valores de pH da solução de $\mathrm{HCl} 0,1 \mathrm{M}(1,0 ; 1,2$ e 1,4$)$ e as concentrações da solução de ácido clorídrico (0,05; 0,1 e 0,2 M).

\section{Análises estatísticas}

A análise estatística dos dados foi realizada através de análise de variância ANOVA unifatorial, onde os resultados são considerados significativos quando a probabilidade é inferior a $5 \%(\mathrm{p}<0,05$, intervalo de confiança de 95\%), teste $t$ de Student com nível de significância $\alpha=0,05$ (intervalo de confiança de 95\%). A avaliação estatística dos resultados foi realizada através do software Microsoft Office Excel ${ }^{\circledR}$, versão 2007. 


\section{Doseamento do CRV matéria-prima e em comprimidos}

O método desenvolvido por espectrofotometria na região do UV foi empregado no doseamento do CRV matéria-prima e em comprimidos. Este foi conduzido em comprimento de onda de 240 $\mathrm{nm}$, empregando-se $\mathrm{HCl} 0,1 \mathrm{M}$ como solvente. As análises por CLAE e por TP foram realizadas conforme os métodos previamente validados para a determinação do teor de CRV matéria-prima e em comprimidos matriciais. ${ }^{20,27}$

\section{Dissolução}

Os comprimidos matriciais contendo $50 \mathrm{mg}$ de CRV foram submetidos ao ensaio de dissolução, utilizando-se aparato II, operado em uma velocidade de $50 \mathrm{rpm}$. O meio de dissolução constituiu-se de $900 \mathrm{~mL}$ de $\mathrm{HCl} 0,1 \mathrm{M}(\mathrm{pH} 1,2)$ a $37^{\circ} \mathrm{C}$. Em intervalos de tempo pré-determinados, alíquotas de $10 \mathrm{~mL}$ foram retiradas, sendo esta quantidade imediatamente reposta com $\mathrm{HCl} 0,1 \mathrm{M}$. As amostras foram centrifugadas e analisadas utilizando-se um espectrofotômetro na região UV em $240 \mathrm{~nm}$.

\section{RESULTADOS E DISCUSSÃO}

\section{Validação do método analítico por espectrofotometria na região do UV}

A validação é uma ferramenta capaz de demonstrar, por meio de estudos experimentais, que o método é apropriado para a finalidade a que se destina, garantindo a confiabilidade dos resultados obtidos. ${ }^{12,15,29,30}$ Dessa forma, este é considerado um dos principais instrumentos dentro da garantia da qualidade de uma formulação farmacêutica. ${ }^{13}$

A especificidade é a capacidade do método em medir exatamente um composto na presença de outros componentes que possam ser esperados em determinada amostra, tais como impurezas, produtos de degradação e componentes da matriz. ${ }^{12,15,29,30}$

De acordo com os espectros apresentados na Figura 2, não ocorreu a sobreposição dos picos referentes ao fármaco e à solução placebo na faixa de absorção máxima do CRV $(240 \mathrm{~nm})$. Desta forma, assegura-se que o método é específico no comprimento de onda utilizado para as análises, não havendo, portanto, interferência dos demais componentes da matriz hidrofílica na quantificação do fármaco.

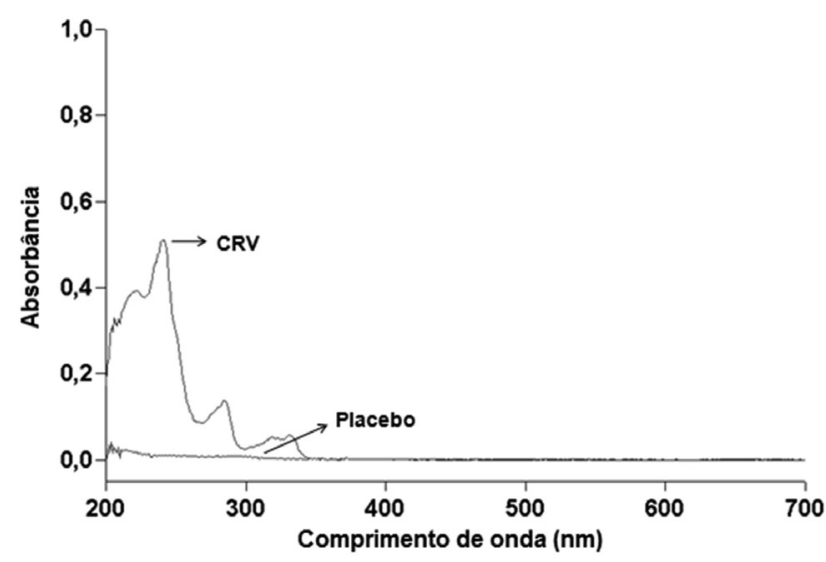

Figura 2. Espectro de absorção no UV-VIS do CRV $\left(6 \mu \mathrm{g} \mathrm{mL}^{-1}\right)$ e solução placebo em $\mathrm{HCl} 0,1 \mathrm{M}$

A linearidade é conceituada como a capacidade de um método analítico em obter resultados diretamente proporcionais à concentração do analito na amostra, dentro de um intervalo específico. ${ }^{12,15,29,30,32}$ Este parâmetro foi mensurado por meio de análises estatísticas de variância, obtendo-se resultados significativos para a regressão linear e não significativos para o desvio da linearidade (p $<0,05)$. O coeficiente de correlação encontrado para a curva média foi de 0,9999 (Tabela 1).

Tabela 1. Dados referentes à linearidade do método

\begin{tabular}{lc}
\hline Parâmetros & Resultados \\
\hline Faixa de linearidade & $2,0-10 \mu \mathrm{g} \mathrm{mL}{ }^{-1}$ \\
Equação: $\mathrm{y}=\mathrm{ax}+\mathrm{b}$ & $\mathrm{y}=0,1045 \mathrm{x}+0,0052$ \\
Inclinação (b) \pm desvio padrão & $0,0052 \pm 1,9860$ \\
Inclinação (a) \pm desvio padrão & $0,1045 \pm 1,7521$ \\
Coeficiente de correlação (r) & 0,9999 \\
\hline
\end{tabular}

A sensibilidade do método foi analisada através da determinação dos limites de quantificação (LQ) e de detecção (LD). O LD é a menor quantidade do analito presente em uma amostra passível de detecção, embora não seja necessariamente quantificado sob as condições estabelecidas pelo método proposto. Em contrapartida, o LQ é a menor quantidade do analito que pode ser determinada com precisão e exatidão aceitáveis sob as condições estabelecidas. ${ }^{12,15,29,30,33}$ Para esses parâmetros os valores calculados foram de 0,5118 e 0,1689 $\mu \mathrm{g} \mathrm{mL} \mathrm{m}^{-1}$, LQ e LD, respectivamente. Analisando-se o valor de LQ, quase quatro vezes menor que a concentração mínima estabelecida no intervalo de quantificação, pode-se constatar que o método proposto apresenta excelente sensibilidade, visto que durante a dissolução/ liberação do fármaco se espera quantificar desde valores muito baixos de concentração até valores mais elevados. Desta forma, assegura-se que o método proposto é capaz de detectar e quantificar uma ampla faixa de concentrações com segurança.

A precisão do método avalia a proximidade dos resultados obtidos em uma amostragem múltipla referente a uma mesma amostra. Os resultados alusivos à repetibilidade (precisão intracorrida) e à precisão intermediária (precisão intercorridas) foram expressos em valores de desvio padrão relativo (DPR). Os resultados da precisão do CRV matéria-prima e em comprimidos matriciais estão apresentados nas Tabelas 2 e 3, respectivamente.

Tabela 2. Resultados da repetibilidade (mesmo dia) e precisão intermediária (dias diferentes) do CRV matéria-prima

\begin{tabular}{llll}
\hline $\begin{array}{l}\text { Solução amostra } \\
\left(6 \mu \mathrm{g} \mathrm{mL} \mathrm{m}^{-1}\right)\end{array}$ & $\begin{array}{l}\text { Absorbância } \pm \\
\text { D.P.R.\% }\end{array}$ & $\begin{array}{l}\text { Solução amostra } \\
\left(6 \mu \mathrm{gL}^{-1}\right)\end{array}$ & $\begin{array}{l}\text { Absorbância } \pm \\
\text { D.P.R.\% }\end{array}$ \\
\hline Manhã & $0,6675 \pm 0,1271$ & Dia 1 & $0,6675 \pm 0,1271$ \\
Tarde & $0,6620 \pm 0,0107$ & Dia 2 & $0,6854 \pm 0,6396$ \\
Noite & $0,6648 \pm 0,4255$ & Dia 3 & $0,6779 \pm 0,9596$ \\
Mesmo dia & $0,6648 \pm 0,4175$ & Dias diferentes & $0,6769 \pm 1,3279$ \\
\hline
\end{tabular}

*Média \pm D.P.R $(\mathrm{n}=6)$.

Para a matéria-prima, a repetibilidade e precisão intermediária apresentaram DPR de 0,42 e 1,33\%, respectivamente. Os valores obtidos contemplam o limite máximo aceito de 5,0\%, conforme recomendação da RE 899, de 29/05/2003.12 A análise estatística de ANOVA demonstrou que não há diferenças significativas entre as análises realizadas nos diferentes períodos, tendo $F$ calculado $\left(F_{\text {cal }}\right.$ $=5,0255)$ e $F$ tabelado $\left(F_{\mathrm{t}}=7,7086\right)$ para $\mathrm{p}<0,05$.

Para os comprimidos matriciais, a repetibilidade e precisão intermediária apresentaram DPR de 0,31 e 0,72\%, respectivamente. Os valores obtidos contemplam o limite máximo aceito de 5,0\%, 
Tabela 3. Resultados da repetibilidade (mesmo dia) e precisão intermediária (dias diferentes) do CRV comprimidos matriciais

\begin{tabular}{llll}
\hline $\begin{array}{l}\text { Solução amostra } \\
\left(6 \mu \mathrm{g} \mathrm{mL}^{-1}\right)\end{array}$ & $\begin{array}{l}\text { Absorbância } \pm \\
\text { D.P.R.\% }\end{array}$ & $\begin{array}{l}\text { Solução amostra } \\
\left(6 \mu \mathrm{g} \mathrm{mL} \mathrm{mL}^{-1}\right)\end{array}$ & $\begin{array}{l}\text { Absorbância } \pm \\
\text { D.P.R.\% }\end{array}$ \\
\hline Manhã & $0,6665 \pm 1,0543$ & Dia 1 & $0,6665 \pm 1,0543$ \\
Tarde & $0,6705 \pm 1,0609$ & Dia 2 & $0,6608 \pm 2,0892$ \\
Noite & $0,6675 \pm 1,1144$ & Dia 3 & $0,6754 \pm 1,1436$ \\
Mesmo dia & $0,6682 \pm 0,3132$ & Dias diferentes & $0,6659 \pm 0,7229$ \\
\hline
\end{tabular}

*Média \pm D.P.R $(\mathrm{n}=6)$.

conforme recomendação da RE 899, de 29/05/2003. ${ }^{12}$ A análise estatística de ANOVA demonstrou que não há diferenças significativas entre as análises realizadas nos diferentes períodos, tendo $F$ calculado $\left(F_{\text {cal }}=0,5594\right)$ e $F$ tabelado $\left(F_{\mathrm{t}}=7,7086\right)$, para p $<0,05$.

No intuito de averiguar a exatidão do método analítico proposto, ou seja, a proximidade dos resultados obtidos pelo método em estudo em relação ao valor verdadeiro, ${ }^{12,15,29,30}$ utilizou-se o método de adição de padrão para a verificação desse parâmetro. A porcentagem média obtida para exatidão foi de $99,30 \% \pm 0,51$, resultado que traduz o valor verdadeiro da amostra (Tabela 4). A partir do teste $t$ de Student, pode-se afirmar que o valor de recuperação encontrado é estatisticamente igual a $100 \%$ para nível de significância $\alpha=0,05, t$ calculado $\left(\mathrm{t}_{\text {cal }}=0,0764\right)$ e $t$ tabelado $\left(\mathrm{t}_{\mathrm{t}}=2,7764\right)$.

Tabela 4. Recuperação do CRV adicionado à formulação analisada pelo método proposto

\begin{tabular}{cccccc}
\hline $\begin{array}{c}\text { Padrão } \\
\text { de CRV } \\
\left(\mu \mathrm{g} \mathrm{mL}^{-1}\right)\end{array}$ & $\begin{array}{c}\text { Concen- } \\
\text { tração de } \\
\text { amostra } \\
\left(\mu \mathrm{g} \mathrm{mL}^{-1}\right)\end{array}$ & $\begin{array}{c}\text { Concentra- } \\
\text { ção de } \\
\text { CRV final } \\
\left(\mu \mathrm{g} \mathrm{mL}^{-1}\right)\end{array}$ & $\begin{array}{c}\text { Absorbância } \\
\text { média }\end{array}$ & $\begin{array}{c} \pm \mathrm{DPR} \\
(\%)\end{array}$ & $\begin{array}{c}\text { Recupera- } \\
\text { ção }(\%)\end{array}$ \\
\hline 2 & 2 & 4 & 0,4051 & 0,9731 & 99,08 \\
4 & 2 & 6 & 0,6113 & 0,8884 & 98,92 \\
8 & 2 & 10 & 1,0346 & 0,7742 & 99,88 \\
\hline
\end{tabular}

* $\mathrm{n}=$ média de 3 determinações, Abs = absorbância, DPR = desvio padrão relativo.

A fim de avaliar a robustez do método analítico, ou seja, a medida da capacidade do método em resistir a pequenas variações dos parâmetros analíticos, ${ }^{12,15,29,30}$ verificou-se o comportamento dos resultados em relação a três variáveis: comprimento de onda, $\mathrm{pH}$ da solução de $\mathrm{HCl}$ 0,1 M e concentração da solução de $\mathrm{HCl}$. Os dados experimentais obtidos para robustez são apresentados na Tabela 5. A análise estatística dos dados, realizada através da ANOVA, demonstrou que as pequenas modificações propostas não influenciaram

Tabela 5. Dados relativos à robustez do método proposto

\begin{tabular}{|c|c|c|c|}
\hline Parâmetros variados & & $\begin{array}{l}\text { Absorbância da } \\
\text { solução amostra }\end{array}$ & $\begin{array}{c}\text { Concentração do } \\
\text { fármaco }(\%)\end{array}$ \\
\hline \multirow{3}{*}{ Comprimento de onda } & 238 & 0,6371 & 100,78 \\
\hline & 240 & 0,6668 & 105,52 \\
\hline & 242 & 0,6719 & 106,33 \\
\hline \multirow{3}{*}{ pH da solução } & 1,0 & 0,6367 & 100,72 \\
\hline & 1,2 & 0,6381 & 100,94 \\
\hline & 1,4 & 0,6434 & 101,79 \\
\hline \multirow{3}{*}{$\begin{array}{l}\text { Concentração da } \\
\text { solução (M) }\end{array}$} & 0,05 & 0,6420 & 101,56 \\
\hline & 0,1 & 0,6429 & 101,71 \\
\hline & 0,2 & 0,6456 & 102,14 \\
\hline
\end{tabular}

significativamente nos resultados obtidos. Valores obtidos através da analise estatística, $F$ calculado $\left(F_{c a l}=2,4924\right)$ e $F$ tabelado $\left(F_{\mathrm{t}}=\right.$ 5,1432 ), para $\mathrm{p}<0,05$.

\section{Comparação da precisão entre métodos}

Um método em desenvolvimento pode ser avaliado comparando-se os seus resultados com os obtidos por outro método já validado, desde que estes tenham sido desenvolvidos para os mesmos fins. ${ }^{34,35}$

$\mathrm{O}$ teor de CRV obtido pela técnica espectrofotométrica foi correlacionado com os resultados de técnicas previamente validadas (CLAE e TP) ${ }^{20,27}$ Os resultados obtidos no doseamento do CRV matéria-prima e na forma de comprimidos matriciais estão apresentados na Tabela 6 .

Tabela 6. Dados obtidos no doseamento do CRV matéria-prima e na forma de comprimidos

\begin{tabular}{lcc}
\hline Técnica & $\begin{array}{c}\text { Média }(\%) \pm \text { D.P.R }(\%) \\
\text { matéria-prima }\end{array}$ & $\begin{array}{c}\text { Média }(\%) \pm \text { D.P.R }(\%) \\
\text { comprimidos matriciais }\end{array}$ \\
\hline Espectrofotometria UV & $100,19 \pm 2,43$ & $103,13 \pm 1,95$ \\
CLAE & $102,85 \pm 2,84$ & $101,78 \pm 1,66$ \\
TP & $100,04 \pm 4,50$ & $108,40 \pm 6,25$ \\
ANOVA & $\mathrm{F}_{\text {cal }}(0,3649), \mathrm{F}_{\mathrm{t}}(9,5521) \mathrm{F}_{\text {cal }}(0,6460), \mathrm{F}_{\mathrm{t}}(9,5521)$ \\
\hline
\end{tabular}

Analisando os resultados de doseamento do CRV matéria-prima, verificou-se que as técnicas analisadas apresentaram resultados muito próximos, demonstrando que os métodos são exatos na quantificação do CRV matéria-prima. A análise estatística dos dados, realizada através da ANOVA, demonstrou que os diferentes métodos empregados no doseamento do CRV matéria-prima não influenciaram significativamente nos resultados obtidos. Valores obtidos através da análise estatística, $F$ calculado $\left(F_{\text {cal }}=0,3649\right)$ e $F$ tabelado $\left(F_{\mathrm{t}}=\right.$ 9,5521), para $\mathrm{p}<0,05$.

Em contrapartida, a análise dos dados obtidos na quantificação do CRV em comprimidos a partir das diferentes técnicas apresenta disparidade. Entretanto, a análise estatística de ANOVA demonstrou que não há diferenças significativas no teor de CRV em comprimidos analisados através das diferentes técnicas, tendo $F$ calculado $\left(F_{\text {cal }}=\right.$ 0,6460), $F$ tabelado $\left(F_{\mathrm{t}}=9,5521\right)$, para $\mathrm{p}<0,05$.

Neste contexto, verificou-se que o método espectrofotométrico em desenvolvimento é equivalente aos demais métodos/técnicas, sendo este exato e preciso tanto na quantificação do CRV matéria-prima, quanto do CRV na forma de comprimidos.

\section{Dissolução}

O ensaio de dissolução in vitro determina o percentual de fármaco liberado de sua forma farmacêutica no meio de dissolução, em período de tempo definido na monografia de cada produto, quando é submetido à ação de aparelhagem específica em determinadas condições experimentais. ${ }^{16}$ Este ensaio é um dos instrumentos mais sensíveis para a avaliação in vitro das propriedades biofarmacêuticas do fármaco em preparações sólidas orais.$^{36} \mathrm{O}$ método proposto no presente trabalho apresenta baixos valores de LQ e LD, possibilitando assim o seu emprego na quantificação do CRV em ensaios de dissolução.

O perfil de liberação dos comprimidos matriciais de $50 \mathrm{mg}$ de CRV foi determinado por $24 \mathrm{~h}$ (Figura 3), sendo demonstrado nesse período a totalidade da liberação do fármaco e a aplicabilidade do método de quantificação proposto para este ensaio. 


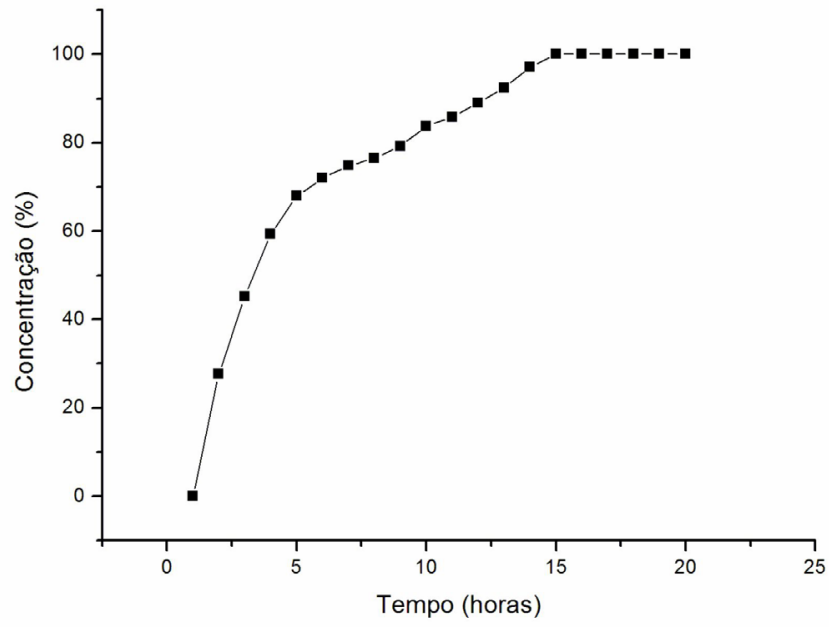

Figura 3. Perfil de liberação do CRV a partir das matrizes hidrofílicas em $\mathrm{HCl} \mathrm{0,1} \mathrm{M}$

\section{CONCLUSÃO}

O método analítico proposto para a quantificação do CRV em matrizes hidrofílicas e na matéria-prima, através da técnica espectrofotométrica na região do UV, mostrou ser específica, linear, precisa, exata e robusta na faixa de quantificação de $2-10 \mu \mathrm{g} \mathrm{mL} \mathrm{mL}^{-1}$. Este também apresentou LQ e LD baixos, que permitem o seu emprego em ensaio de dissolução. Através da análise de ANOVA, aplicada no estudo comparativo, evidenciou-se a ausência de diferenças significativas entre os resultados obtidos através dos diferentes métodos/técnicas analíticas tanto para a determinação de teor do CRV matéria-prima, quanto em comprimidos matriciais. Desse modo, o método espectrofotométrico UV proposto para a quantificação de CRV mostrou-se equivalente aos métodos de CLAE e TP na determinação de teor de CRV matéria-prima e em comprimidos matriciais. Assim, o método validado no presente estudo destaca-se devido à sua rapidez, fácil interpretação, baixo custo operacional, elevada confiabilidade de resultados e aplicabilidade tanto na quantificação da matéria-prima quanto em comprimidos matriciais.

\section{AGRADECIMENTOS}

Ao CNPq/CAPES pelo suporte financeiro e à Indústria Farmacêutica Prati Donaduzzi Ltda por disponibilizar os equipamentos e a infraestrutura necessários para realização de parte deste trabalho.

\section{REFERÊNCIAS}

1. McCarty, R.; Neurosci. Biobehav. Rev. 1983, 7, 493.

2. Sukor, N.; Eur. J. Int. Med. 2011, 22, 433.

3. Stafylas, P. C.; Sarafidis, P. A.; Vasc. Health Risk Manag. 2008, 4, 23.

4. Genaro, A. R.; The science and practice of pharmacy, $20^{\text {th }}$ ed., Lippincott Williams \& Wilkins: Baltimore, 2000.

5. Brophy, J. M.; Joseph, L.; Rouleau, J. L.; Ann. Int. Med. 2001, 134, 550.

6. Hirlekar, R.; Kadam, V.; J. Incl. Phenom. Macrocycl. Chem. 2009, 63, 219.

7. http://www-thomsonhc-com.ez46.periodicos.capes.gov.br/micromedex $2 /$ librarian/ND_T/evidencexpert/ND_PR/evidencexpert/CS/8E501E/ND_ AppProduct/evidencexpert/DUPLICATIONSHIELDSYNC/7F29DE/
ND_PG/evidencexpert/ND_B/evidencexpert/ND_P/evidencexpert/ PFActionId/evidencexpert.IntermediateToDocumentLink?docId=18917n\&contentSetId=30\&title $=$ Carvedilol $\&$ servicesTitle $=$ Carvedilol\& topic Id=null, acessada em Fevereiro de 2013.

8. Lakshmi, M. S.; Sriranjani, M.; Bakrudeen, H. B.; Kannan, A. S.; Mandal, A. B.; Reddy, B. S. R.; Appl. Clay Sci. 2010, 48, 589.

9. Loftsson, T.; Vogensen, S. B.; Desbos, C.; Jansook, P.; AAPS Pharm. Sci. Technol. 2008, 9, 425.

10. Colombo, P.; Bettini, R.; Santi, P.; De Ascentiis, A.; Peppas, N. A.; J. Control. Release 1996, 39, 231.

11. Barros, C. B.; Rev. Biológico 2002, 64, 175.

12. Brasil, Ministério da Saúde, ANVISA - Agência Nacional de Vigilância Sanitária; RE no 899, de 29/05/2003, Guia para validação de métodos analíticos e bioanalíticos, MS: Brasília, 2003.

13. Pereira, A.; Scheshowitsch, K.; Silva, A.; Silva, M. A. S.; Stulzer, H. K.; Visão Acadêmica-Curitiba 2007, 8, 2.

14. British Pharmacopoeia, $5^{\text {th }}$ ed., HM Stacionary Office: London, 2007.

15. The United States Pharmacopeia (USP); $34^{\text {th }}$ ed., United States Pharmacopoeial Convention: Rockville, 2011.

16. Farmacopeia Brasileira, $4^{\mathrm{a}}$ ed., Atheneu: São Paulo, 2002.

17. Galanopoulou, O.; Rozou, S.; Antoniadou-Vyza, E.; J. Pharm. Biomed. Anal. 2008, 48, 70.

18. Sripalakit, P.; Kaewnok, S.; Tubtonglang, S.; Maejo Int. J. Sci. Technol. 2010, 4,8 .

19. Theivarasu, C.; Ghosh, S.; Indumathi, T.; Asian J. Pharm. Clin. Res. 2010, 3,4 .

20. Lanzanova, F. A.; Argenta, D.; Arend, M. Z.; Brum, L. B.; Cardoso, S. G.; J. Liq. Chromatogr. Relat. Technol. 2009, 18, 526.

21. Friedrich, R. B.; Ravanello, A.; Cichota, L. C.; Rolim, C. M. B.; Beck, R. C. R.; Quim. Nova 2009, 32, 1052.

22. Favoretto, L. B.; Souza, J. M. O.; Bonfilio, R.; Azevedo, R. C. P.; Araújo, M. B.; Quim. Nova 2010, 33, 1585.

23. Barboza, F. M.; Vecchia, D. D.; Pereira, A. V.; Silva, M. A. S.; Stulzer, H. K.; Quim. Nova 2010, 33, 747.

24. Alves, L. D. S.; Rolim, L. A.; Fontes, D. A. F.; Rolim-Neto, P. J.; Soares, M. F. L. R.; Soares Sobrinho, J. L.; Quim. Nova 2010, 33, 1967.

25. Görög, S.; Anal. Sci. 2004, 20, 767.

26. Abdelwahab, N. S.; Arabian J. Chem. (2011), doi:10.1016/j. arabjc.2011.05.002.

27. Ieggli, C. V.; Cardoso, S. G.; Belle, L. P.; J. AOAC Int. 2005, 88, 1299.

28. Barboza, F. M.; Stulzer, H. K.; Lat. Am. J. Pharm. 2011, 30, 1202.

29. ICH - International Conference on Harmonization of Technical Requeriments for Registration of Pharmaceuticals for Human Use; Q 2(R1) Validation of Analytical procedures: text and methodology, 2005.

30. ICH - International Conference on Harmonization of Technical Requeriments for registration of Pharmaceutical for Human Use; Q 2B - Guideline on validation of Analytical procedure: methodology, 2005.

31. Rowe, R. C.; Sheskey, P. J.; Owen, S. C.; Handbook of Pharmaceutical Excipients, $5^{\text {th }}$ ed., Pharmaceutical Press: London, 2006.

32. Costa, M. A. B.; Ricci-Júnior, E.; Santos, E. P.; Mansur, C. R. E.; Campos, V. E. B.; Quim. Nova 2012, 35, 808.

33. Tagliari, M. P.; Granada, A.; Kuminek, G.; Stulzer, H. K.; Silva, M. A. S.; Quim. Nova 2012, 35, 1228.

34. EAL - European cooperation for Accreditation of Laboratories; P11 Validation of Test Methods - General principles and concepts, 1997.

35. Brasil, INMETRO - Instituto Nacional de Metrologia: Normatização e Qualidade Industrial; Orientações sobre Validação de Métodos de Ensaios Químicos; DOQ-CGCRE-008, Revisão: Março de 2003.

36. Arancibia, A.; Acta Farm. Bonaerense 1991, 10, 123. 